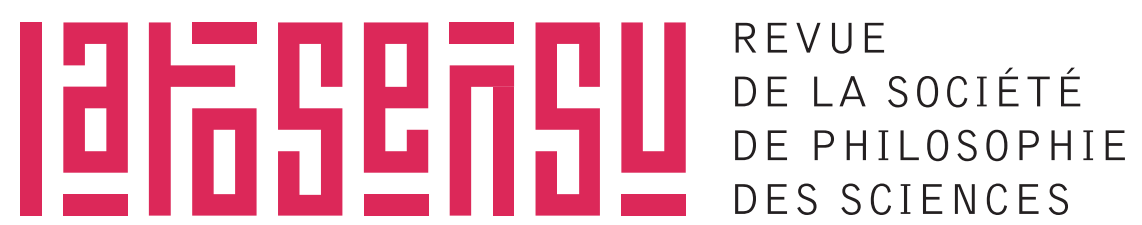

Vol $5 N^{\circ} 12018$

http://dx.doi.org/10.20416/lsrsps.v5i1.10

Valia Allori

Scientific Realism

and Primitive Onto-

LOGY OR:

The Pessimistic In-

DUCTION AND THE NA-

TURE OF THE WAVE

FunCTION

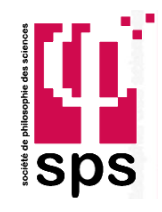

SOCIÉTÉ DE PHILOSOPHIE DES SCIENCES (SPS) 


\section{Valia Allori}

\section{Scientific Realism and Primitive Ontol- ogy Or: The Pessimistic Induction And the Nature of the Wave Function}

\author{
Sommaire \\ 1 - Introduction \\ 2 - The Pessimistic Meta \\ Induction and Explanatio- \\ nism \\ 3 - The Classical-to-Quantum \\ Theory Change as a Problem \\ for Explanationism \\ 4 - Primitive Ontology \\ 5 - Quantum Mechanics and \\ Explanationism \\ 6 - Possible Objections and \\ Replies \\ 7-An Argument for the PO \\ Approach over Wave Function \\ Realism based on Scientific
} Realism
In this paper I wish to connect the recent debate in the philosophy of quantum mechanics concerning the nature of the wave function to the historical debate in the philosophy of science regarding the tenability of scientific realism. Advocating realism about quantum mechanics is particularly challenging when focusing on the wave function. According to the wave function ontology approach, the wave function is a concrete physical entity. In contrast, according to an alternative viewpoint, namely the primitive ontology approach, the wave function does not represent physical objects. In this paper, I argue that the primitive ontology approach can naturally be interpreted as an instance of the so-called explanationist realism, which has been proposed as a response to the pessimistic-meta induction argument against scientific realism. If my arguments are sound, then one could conclude that: (1) contrary to what is commonly thought, if explanationism realism is a good response to the pessimistic-meta induction argument, it can be straightforwardly extended also to the quantum domain; (2) the primitive ontology approach is in better shape than the wave function ontology approach in resisting the pessimistic-meta induction argument against scientific realism.

\section{1 - Introduction}

Scientific realism would be a commonsensical philosophical position if there weren't powerful counter-arguments to it, the most famous of which is the pessimistic meta-induction (PMI) argument: since past successful theories turned out to be false, it is unwarranted to believe that our best current theories are true simply because they are successful (Laudan 1981). Some scientific realists have responded to the PMI argument by restricting realism to a subset of the theoretical entities of the theory. One particular way of doing this is explanationist realism (or explanationism), according to which one should be a realist with respect to the working posits of the theory, the ones involved in explanations and predictions. In contrast, one does not need to commit herself to believe in the existence of other presuppositional posits of the theory, since they are somewhat 'idle' components (Kitcher 1995; Psillos 1999). The proponents of this view have argued for it in the framework of classical theories (e.g. Fresnel's theory of light). I think the case for explanationism is fundamentally incomplete if one does not consider the theory change from classical to quantum mechanics. In this paper, I argue that explanationism can be extended to the quantum framework. In order to show this, I discuss the different realist approaches to quantum mechanics, which differ in the interpretation of one of the fundamental objects of the theory: the wave function. On the one hand, according to the wave function ontology approach, also known as wave function realism, the wave function is a concrete physical entity (Albert 1996; Loewer 1996; Lewis 2004, 2005, 2006; Ney 2012, 2013). In contrast, according to the primitive ontology (PO) approach (Allori et al. 2008; Allori 2013), the wave function does not represent physical objects. I argue that the PO approach can provide what explanationism needs to defeat the PMI argument in the classical-to-quantum transition. The PO, and not the wave function, can be identified with the working posit of quantum theory, and as such: (1) it is primarily responsible for the success of both classical and quantum mechanics; and (2) it is (suitably) preserved in the classical-to-quantum theory change. Notice that explanationism so understood provides an argument in favor of the PO approach over the wave function ontology approach: being preserved in theory change, the PO defeats the PMI argument. In contrast, since the wave function does not have any classical analog, if wave function realism is true it is hard to see how the working posits are preserved in the transition from classical to quantum mechanics. Thus, wave function realism may undermine scientific realism about quantum mechanics.

The paper is structured as follows: in the next section, I overview the PMI argument against scientific realism and some replies to it, focusing on explanationism. Then in Section 3 I explain how the transition from classical to quantum mechanics is a problem for explanationism. In Sections 4 and 5 I present the PO approach as a framework to understand fun- 
damental physical theories, I show how the PO is preserved through the classical-to-quantum theory change and I argue that it is the PO, rather than the wave function, which is responsible for the empirical success of quantum mechanics. In Section 7 I discuss some objections to the previous discussion, and I provide some replies. Finally, in the last section I discuss the advantage of the PO approach over the wave function realism approach in responding to the PMI argument.

\section{2 - The Pessimistic Meta-In- duction and Explanationism}

Scientific realism is, roughly put, the view that scientific theories give us a (nearly) truthful description of the world. The main argument for scientific realism, the no-miracle argument, holds that the empirical success of a theory can, and should, be taken as evidence of its truth. Nonetheless, there are very powerful arguments against scientific realism, such as the pessimistic meta-induction (PMI), argument. The main idea of the PMI is to go against the no-miracle argument. The claim is that it is not the case that the empirical success of a theory is a reliable indicator of its truth: since past theories were empirically successful but turned out to be false, it follows that our current theories, even if successful, are more likely to be false than true.

One way to respond to the PMI challenge is to argue that one should be a realist about a restricted set of entities, not about the whole theory. This is what Psillos calls a divide et impera strategy: scientific realists may argue that "when a theory is abandoned, its theoretical constituents, i.e. the theoretical mechanisms and laws posited, should not be rejected en bloc. Some of those theoretical constituents are inconsistent with what we now accept, and therefore they have to be rejected. But not all are. Some of them have been retained as essential constituents of subsequent theories" (Psillos 1999, p. 108). Therefore, if one can show that the entities that are retained in moving from one theory to the next are the ones that are responsible for the empirical success of the theory, the PMI is blocked. By restricting realism one provides an alternative explanation for the success of past false-but-successful theories: past theories were successful not because they were (approximately) true in their entirety, but because some parts of them were. If these true constituents of past theories are responsible for the theories' success and they are carried over in theory change, then we are justified in believing that the entities these theoretical constituents represent exist in reality.

There are various ways to restrict realism. One example is Worrall's structural realism (Worrall 1989), according to which the PMI is correct in saying that in theory change we often have discontinuity at the level of unobservable entities, but most of the mathematical content of the old theory carries over to the new one. Therefore, the scientific realist may not be justified in believing what the theory says about the nature of physical objects. Nonetheless she is justified in believing that the structure that holds between these objects which is preserved in theory change is (approximately) true. There are different varieties of structural realism, a first rough distinction is the one between epistemic structural re- alism and ontic structural realism. In the epistemic version, which some attribute to Worrall himself, we are only justified in believing that objects stand in certain structural relations with one another. Ontic structural realism instead goes further and claims that the very notion of objects is problematic and is worth dismissing (French 1998; Ladyman 1998). There are other responses to the PMI argument, but in this paper, I will focus on explanationism, developed most prominently by Kitcher (1993) and Psillos (1999). They distinguish between 'working' and 'presuppositional' posits of a theory. The working posits are the ones that are responsible for the theory's empirical success, while the presuppositional posits are theoretical constituents needed to complete the theory. If the working posits are preserved during theory change, the argument goes, one could argue that past theories were successful because they got something right, namely the working posits. At the same time, when considered in their entirety, these theories are also false since they got something wrong too, namely the presuppositional posits. Thus, the advocate of realism is justified in believing in the reality of the working posits, but there is no need for her to commit to the existence of the presuppositional posits: they are just 'idle' components, which make no difference to the theory's success.

\section{3 - The Classical-to-Quantum Theory Change as a Problem for Explanationism}

Scientific realism has been motivated and discussed almost exclusively in the classical framework, discussing theories other than quantum mechanics. In particular, Psillos and Kitcher argue for explanationism within Fresnel's theory of light. It was successful because it got the working posit right, namely the electromagnetic waves: they are responsible for the success of the theory, and they were preserved by Maxwell's electrodynamics. In contrast, ether was a presuppositional posit: the success of Fresnel's theory did not depend on it, and it was abandoned by the subsequent theory. Realists are therefore justified in believing that electromagnetic waves exist, but do not have to be committed to believe that ether exists too. Another example extensively discussed in the literature is the caloric theory of heat, or phlogiston's theory of combustion, to again arrive at the conclusion that caloric and phlogiston are presuppositional posits. In reply, these historical examples have been revisited with the intent of arguing that ether, caloric, phlogiston, and the like, contrary to what it is maintained by explanationism, played an important role in the success of past theories (see, e.g. Psillos (1994), Chang (2003), Elsamahi (2005)).

Regardless of the outcome of the debate over these examples, I think that the main threat to explanationism comes from the classical-to-quantum transition. The fact that the discussion was limited to classical theories is not surprising: quantum mechanics has been considered, for a long time, incompatible with scientific realism. While, on the one hand, quantum theory is incredibly powerful in making new and very precise predictions, on the other hand it is extremely difficult to understand and 'make sense' of. Indeed, quantum mechanics 


\section{SCIENTIFIC REALISM AND Primitive Ontology Or: The Pessimistic IN- DUCTION AND THE NATURE of THE WAVE Function}

has been taken by many to suggest that physical objects have contradictory properties, like being in a place and not being in that place at the same time, or that properties do not exist at all independently of observation. Given that, many have thought that the real lesson of quantum mechanics is that scientific realism is untenable. Luckily for the realist ,the situation has changed: today we have various proposals of quantum theories that allow for a realist reading. Among these theories, most famously, we find Bohmian mechanics (Bohm 1952), Everettian quantum mechanics , or many-worlds theory (Everett 1957), and the GRW theory (Ghirardi, Rimini \& Weber 1986). They are empirically adequate fundamental quantum theories according to which there is an objective physical world, which can be described by non-contradictory, mind-independent properties.

Nonetheless, even in this framework the examples from quantum mechanics are brought up to motivate ontic structural realism rather than explanationism: "[what] we have learned from contemporary physics is that the nature of space, time and matter are not compatible with standard metaphysical views about the ontological relationship between individuals, intrinsic properties and relations" (Ladyman 2014). Most importantly, in quantum mechanics we have the Schrödinger equation, which is the equation for the temporal evolution of the wave function. The wave function is an object which is involved in the derivation of most, if not all, predictions and explanations the theory is able to provide. Thus, in the framework provided by explanationism, it seems one should regard the wave function as a working posit of quantum theory. If so, though, explanationist realism is not able to defeat the PMI, since the wave function arguably does not have any classical analog and therefore we have radical discontinuity in the classical-to-quantum theory change. This is bad news, since the case for explanationism has no hope of being compelling if it does not cover quantum mechanics. In the next section, though, I show how explanationist realism can be extended to quantum theories if paired with a particular view about the metaphysics of quantum mechanics, namely the primitive ontology (PO) approach.

\section{4 - Primitive Ontology}

Most philosophers of physics recognize the legitimacy of Bohmian mechanics, Everettian theory and GRW, but disagree about the metaphysical pictures these theories provide. In this section, I discuss the PO approach. In the next section I argue that all these theories have a primitive ontology such that: (1) it is primarily responsible for the theory's success; and (2) it (suitably) carries over during theory change. If so, and assuming that a strategy like explanationism is successful in defending scientific realism, the PMI is blocked: the advocate of realism is justified in believing that the $\mathrm{PO}$ is real because it does all the work to explain the empirical success of theories and it is (suitably) preserved in theory change.

Here is a brief summary of the PO account (Allori et al. 2008; Allori 2013). Arguably, classical theories such as Newtonian mechanics, classical electrodynamics and statistical mechanics share a common way of understanding how fundamental physical theories account for the behavior of macroscopic phenomena. The reason is that all these theories possess a PO: some variable in three-dimensional space (or four-dimensional space-time) that represent physical objects. For classical theories, the PO was point-particles. ${ }^{1}$ If we wish to preserve such a common framework, then any other fundamental physical theory should have a PO as well. The primitive variables in the quantum theories proposed so far in the literature include point-particles, continuous fields, and spatio-temporal events or 'flashes.' For instance, Bohmian mechanics is a theory with a particle PO (Dürr, Goldstein and Zanghí 1992), GRWm is a theory in which matter is described by a continuous (three-dimensional) matter field localized where the macroscopic objects are (Benatti, Ghirardi and Grassi 1995), while GRWf is a theory of 'flashes,' spatio-temporal events (Bell 1987, Tumulka 2006). ${ }^{2}$ In addition of specifying what matter is, we need to specify how it behaves. This is implemented by the so-called non-primitive variables, most importantly by the wave function. The fundamental idea is that the wave function does not represent material things, but rather determines how material things move. On the one hand, the wave function is a necessary ingredient to implement the law of temporal evolution of the PO (Allori 2013). For instance, in Bohmian mechanics the wave function evolves according to the Schrödinger equation, while in GRWf and GRWm it evolves according to the Schrödinger equation and then randomly collapses, following the so-called GRW evolution. In Bohmian mechanics the wave function generates the spatio-temporal trajectories of the PO through an equation appropriately dubbed the guide equation. Similarly, in GRWm, with a different equation, the wave function generates the spatio-temporal behavior of the mass-density field. In GRWf, the wave function enters in the probability distribution of the flashes, and therefore determines where they are located in space-time. In this sense, the wave function is involved in the predictions and in the explanation of the phenomena. Nonetheless, on the other hand, the PO is the one that makes direct contact with the phenomena and is ultimately responsible for explanations and predictions. In fact, one of the important features of the PO approach is its architectural component: on the foundation there is the PO, the building block of everything else, and then there are layers of other entities, each of which acquires its significance and its role in the theory via the way they affect the PO. They contribute generating the empirically adequate trajectories of the PO which ultimately play the explanatory role. The wave function should be thought as belonging to this latter category of entities: it helps the PO to explain and to predict, but it is not ultimately responsible for these things.

Here are some fundamental features of the PO approach that is crucial to articulate:

1. REDUCTIONISM with respect to the PO. In this approach, macroscopic objects are thought to be fundamentally composed of the microscopic entities the PO specifies. As such, the PO approach is (ontologically) reductionist, at least to the extent that it allows to make sense of claims like the PO being "the building blocks of everything else," and of the idea that macroscopic regularities are obtained entirely from the microscopic tra- 
jectories of the PO.

2. EXPLANATION and PO. The PO explains the macroscopic regularities using reductionist approaches similar to those used in classical mechanics. In fact, in classical mechanics, macroscopic bodies are made of a collection of particles, and their properties are accounted for in terms of the interaction of these particles among each other and the particles of the environment. For instance, the transparency of a pair of glasses is explained in terms of the electromagnetic forces acting between the particles composing the glasses, which are such that incoming light rays will pass through them (Halliday and Resnick 1962). Similarly, the PO grounds the explanatory schema of quantum theories: the properties of macroscopic objects are (in principle) accounted for in terms of the PO's behavior (Allori 2013).

3. THEOREITICAL VIRTUES. The PO of a theory is postulated, rather than inferred from the formalism, on the basis of some super-empirical virtues such as simplicity, explanatory power, and unification. The PO that provides the simplest, most unifying explanation should be selected (Allori 2015).

4. UNDERDETERMINATION of the WAVE FUNCTION. The way the wave function evolves in time is irrelevant as long as the law of the PO such a wave function defines remains the same. That is, a theory of particles which follow certain trajectories, such as Bohmian mechanics, can be obtained by a Schrödinger-evolving wave function, as in the usual formulation, but also in terms of a collapsed wave function (Allori et al. 2008). Two theories with the same trajectories for the PO, regardless of how they have been obtained (i.e., via a Schrödinger evolving wave function or not) are physically equivalent. Since different wave functions can give rise to the same trajectories for the PO, and since the trajectories of the PO are the ones that account for the macroscopic regularities, the wave function evolution is underdetermined by the data. Because of this, the same trajectories of the PO do not uniquely pick out a wave function. Indeed, it is possible to try to eliminate the wave function completely, as some have tried (Dowker and Herbauts 2006; Norsen 2010).

5. PREDICTIONS and PO. Once the PO and its law of evolution have been chosen, everything else is determined, including the empirical predictions which are determined as a function of the PO. The wave function appears into the derivation of the predictions of the theory, but its role is not essential, since the way in which it specifies the law of the PO is underdetermined (Allori et al. 2014).

\section{5 - Quantum Mechanics and Explanationism}

I wish to argue now that the PO can be identified with the working posit of quantum mechanics, while the wave function is best seen as a presuppositional posit. In fact, as discussed in (5) in the list in the previous section, the predictions are determined by the PO, not by the wave function. It appears in the derivation but its evolution is underdetermined by data, as argued in (4). In addition, as pointed out in (2), explana- tion is in terms of the PO: this reminds one of Kitcher's idea that working posits are the entities that play a fundamental role in the theory's explanatory schemata. Moreover, there is the explicit fundamental postulation that the PO represent matter, while the wave function does not, and that everything is made of the entities the PO specifies, as outlined in (1). All primitive ontologists (or supporters of suitably related views) maintain that one should be a realist about the PO, regardless of what they think the wave function really is. In fact, it has been considered to be, among other things, a law-like object (Goldstein and Zanghí 2013), a disposition (Esfeld et al. 2014), a property (Monton 2006), or a new kind entity (Maudlin 2013). Some others have denied, in line with the Humean tradition, an ontological status of the wave function, taking it to be a parameter that supervenes on the Humean mosaic that is provided by the PO (Bhogal \& Perry 2015; Callender 2015; Esfeld 2014, Miller 2014). Nonetheless, I ague, one can be 'metaphysically neutral' with respect to the wave function: one does not need to commit to the existence or non-existence of the wave function in order to account for the success of the theory. But this is to say that the PO is a working posit, while the wave function is a presuppositional posit of quantum theories. If so, the PO approach provides a very nice framework for the explanationist to extend her view in the quantum domain.

Assuming the arguments in the previous sections are sound, we have made good progress. However, this is not enough to successfully reply to the PMI: one would also have to show that the PO is preserved during theory change. What is the PO of classical mechanics? Arguably, in classical mechanics matter is made of particles, objects with the fundamental property of having a position in three-dimensional space. Therefore, for quantum theories of particles like Bohmian mechanics, the preservation of $\mathrm{PO}$ during the classical-to-quantum theory change is obvious. The interesting cases are the ones that involve a matter density PO and a flash PO. In both cases, literally, the PO of classical mechanics has not straightforwardly carried over.

There seems to be two options: either dispute that these are cases of radical discontinuity, or take this to be an argument against a non-particle PO. I think the latter: particles are the simplest PO, and I do not see why one would make things complicated if there is no need. If you add to this that particles make it easier to defeat the PMI, then the case is even more straightforward. Nonetheless, some may argue that is not true that a particle PO is not enough mentioning that 'flashy' theories like GRWf are more compatible with relativity than particle theories like Bohmian mechanics (Tumulka 2006). I think this is not necessarily the case, as relativistic, non-artificial particle quantum theories have been proposed (Dürr et al. 1998). Be that as it may, one could also argue that the classical-to-quantum change in PO from particles to non-particles is not a case of radical discontinuity after all. In fact, while it is not true that there are particles, it is still the case that there is 'stuff' in three-dimensional space. In other words, we did not bet correctly on the nature of objects in classical mechanics since we believed there were particles while actually there are not. Nonetheless, the assumption that there are three-dimensional entities is left unaffected, 
and there is a sense in which this non-particle three-dimensional 'stuff' behave as if it were a particle. Let me elaborate. Consider the case in which people went from believing that atoms are indivisible to believe that they are made of other indivisible particles. The old theory got right that there are particles, but it was wrong about what the fundamental particles really were. The situation here is different: we move from a theory in which the fundamental entities are particles, to a theory in which the fundamental entities have another nature. This is, arguably, what would happen from a quantum theory of particles to string theory. In this case we are not getting the nature of the fundamental entities right: before we had one-dimensional particles, now we have bi-dimensional vibrating loops. However, if we 'squint,' then we don't see the fine-grained details, and we take strings to be particles. They are, for all explanatory purposes, particles: we explain the macroscopic regularities in terms of the PO, namely three-dimensional 'stuff', ignoring the details about what its real nature actually is. This is similar to the case in which we observe a hose from a distance and we think it is one-dimensional while it is actually two-dimensional, or when we look at a poster in the subway and we think it's a homogenous image when it is actually a collection of colored dots. At the level of microphysics we may have flashes or a continuous field, but at some mesoscopic level they produce trajectories as if they are produced by particles. So, even if the microscopic PO is not one of particles, there is a mesoscopic scale in which they behave as if they are in the sense that from that level up to the macroscopic level the explanation is the same as if they were particles.

Notice that this option is not available to the wave function realist. In fact, one might think that she could argue along the very same lines I just did by saying: "we do not get the nature of objects right because we believe they are composed of particles in classical mechanics and then we discover they are actually, say, patterns in the wave function; but we get something right, namely that on some mesoscopic level they behave as if their nature were the one of particles." The disanalogy between this and my argument is that in the latter what is crucial is the existence of microscopic patterns at the fundamental level in three-dimensional space. It is only because the fundamental objects described by the PO are in such space that we can 'squint' and recover the familiar macroscopic world. In contrast, in the world described by wave function realism one needs to do much more than mere 'squinting.' Indeed, it is unclear what is necessary to do from the highly-dimensional fundamental space to the three-dimensional world of our experiences: different approaches have been proposed, and they are all works in progress (see most notably (Albert 2013, 2015); (Lewis 2013); (Ney 2015, 2017, forthcoming); (North 2013)). In connection to this, a well-known argument against wave function realism is the so-called macro-object problem, namely that wave function realism cannot account for three-dimensional objects (Monton 2002; Maudlin 2007; Allori 2013). Note that my remark is different: even if the wave function realist would be able to account for them, the explanation would have to rely on totally new mechanisms. In fact, while the PO approach can rely on compositionality and reductionism techniques, this is not the case within wave function realism (see Allori (2013) for a similar remark, and Ney (2017) and reference therein for a presentation of some of the new strategies and what is required by them).

\section{6 - Possible Objections and Replies}

One could object that it is wrongheaded to think the wave function is not a working posit of quantum mechanics. In fact, in Kitcher's characterization, the working posits are the ones that are indispensable for the explanation of the phenomena. Therefore, one may think that the wave function is exactly that: it is needed to make contact with the experimental predictions just as well as the PO. It helps explain why the particle, say, goes this way rather than that way in a magnetic field just as well as the PO. Hence, the wave function is not a working posit but a presuppositional posit. ${ }^{3}$ As a reply, I think the correct way to think about the wave function is that it is not needed in the same way as the PO, neither to make contact with the phenomena nor to explain them. In fact, remember the architectural structure of the PO approach: in contrast with the PO, the wave function is not at the foundation of a theory and, even if it helps the PO explain and predict, it is not what is ultimately responsible for these things.

Another objection could be that the chosen example makes things too easy for the PO approach. In fact, the particle PO is carried over from classical to quantum mechanics by construction, so the question I explore is either not very interesting or has no great significance. In contrast, I should ask what is the PO of, say, phlogiston theory and give a good reason why the PO of phlogiston theory does not include phlogiston. ${ }^{4}$ The same could be true for ether as well as for caloric. The issue has been discussed without reference to the $\mathrm{PO}$ and, as already pointed out in Section 3, people have argued that ether, caloric and phlogiston are not among the working posits of the theory. If these arguments are convincing, then one may safely claim that ether, caloric and phlogiston are not the PO of these theories either. To elaborate a little, one may point out that, as Psillos (1994) does, the caloric theory of heat does not contain the material representation of heat as a working hypothesis. Rather, what is relevant is the quantity of heat, which does not need to have any referent. In addition, Psillos denies the holistic theory of confirmation according to which all assumptions of a theory are equally likely to be true. In this way, even if caloric theory was successful and regarded as true, it did not imply that caloric had necessarily to exist as a material entity. Phlogiston theory presupposed the existence of an element, phlogiston, contained within bodies and released during combustion. As it is known, it was replaced by oxidation theory, according to which combustion gives out nothing, but rather oxygen is taken in. I will not pursue here historical research, like Psillos did for caloric, regarding whether contemporary scientists were cautious or not regarding the existence of a material phlogiston. Be that as it may, it seems to me that what was argued for caloric applies here too: what matters is that something was exchanged, and experiments later revealed it was taken in rather than given out, 
as postulated by the phlogiston theory. In the case of ether, the situation is slightly more complicated but analysis leads to the result that ether is a working posit. One may think of classical electrodynamics as a theory with different possible POs (Allori 2015). Among them, classical electrodynamics could be taken to be a theory with a particle PO where the electromagnetic fields are treated as non-primitive variables. In this case ether, as well as the electromagnetic fields, is by definition a working posit, namely something that is needed to account for the phenomena but never the phenomena themselves. Alternatively, one could think of classical fields as part of the PO together with the charged particles, and also in this case ether would just be part of the ingredients to explain macroscopic regularities, and thus a working posit. Thus, it seems unwarranted to think of caloric, phlogiston or ether as part of the PO of the respective theories: the PO is given by the relevant theory, namely Newtonian mechanics or classical electrodynamics. Then the behavior of such PO is refined by experiments and formalized in subsequent theories: kinetic theory, oxygen theory and relativity.

In addition, an anonymous referee has suggested that 'flashy' theories are not so easy to accommodate in the squinting argument I elaborated above, since there may be situations in which no amount of squinting will allow us to recover particle-like behavior from the behavior of flashes. The referee gives the example of a beta-particle in a cloud chamber. According to GRWf, the beta-particle itself will on average flash only once in $10^{8}$ years. The 'particle' track is accounted for by flashes of the surrounding gas particles. Then according to the referee one could conclude that "if the beta particle is to play any role in this explanation (as it does in the classical picture), it is entirely due to its wave function; its flashes (even in the very improbable case that there is one) are completely irrelevant." I believe this conclusion is unwarranted and seems to rely on missing some crucial ingredient of the PO account. In fact, the flashes are not irrelevant: they are the things drawing the track of the beta-particle. The wave function determines the distribution of the flashes in space-time, and as such contribute to explain why the track looks one way rather than another. However one should not overestimate this explanation, which would be empty without the PO.

One, perhaps more interesting observation is the following: isn't my proposal just some sort of (ontic) structuralism? If we do not preserve the nature of 'stuff,' isn't what we preserve some structural content of the theory? In replying to this, let me first make a clarification: if structuralism is the view that there is just structure and no objects as individuals, then clearly not, since the PO approach postulates the existence of objects as a starting point. Instead, what about a moderate version of ontic structural realism, like the one proposed by Esfeld (2004)? The idea behind this view is something like this: one should be a realist about structure but, in contrast with the 'eliminativist' ontic structuralism mentioned above, there are 'things' (individuals) that stand in the relation the structure prescribes, even if they have no intrinsic identities. In the quantum domain, such structure is the wave function. Indeed, interestingly enough, Esfeld (2017) proposes that in his moderate ontic structuralism, the relata the wave function relates are given by the PO: he argues that the PO ap- proach and his moderate ontic structuralism can help each other make sense of quantum non-locality and entanglement. So, in his view, one should be a realist about the PO, and also about the structure that relates the PO, provided by the wave function. In this sense, the reading I provide of the PO approach is different: the strength of the PO approach in responding to the PMI is that it regards the wave function as a working posit. Only because of this, one can show there is continuity of PO during theory change. Instead, Esfeld's moderate ontic structuralism in this 'flat' reading I have just provided does not have this advantage: if the wave function is the structure the advocate of realism should be committing to, then it is difficult to see where this structure was coming from in classical physics. In light of the fact that Esfeld (2014) has previously argued that the wave function should be just considered as part of the Humean mosaic, I think the best way to think about this approach is to emphasize the architectural component of the PO approach and re-read Esfeld's moderate structuralism as non-flat. That is, one would maintain that the PO constitutes the foundation of the theory, while the relations (in which the wave function plays an important role) generate the macroscopic appearances through the PO. In this way, since the focus is on the PO, one need not think of the wave function as a working posit, and thus defeat the PMI.

Let me close this section pointing out how the PO approach can help reply to one common objection to explanationism as originally proposed. The charge is that the distinction between working posits and presuppositional posits can be drawn only after one discovers that the original theory is false. That is, the working posits are what we see have carried over (see, e.g. Stanford (2003a, 2003b)), and this is problematic because if one does not know what the presuppositional posits of the theory are, then one does not know what she is justified in believing. In the PO framework this is not true. In fact, the PO is postulated when the theory is proposed, rather than inferred from the formalism or selected post-hoc: it is the one that provides the best combination of simplicity, explanatory power and unification that is able to account for the experimental data. In this way, the working posits are selected at the time the theory is proposed.

\section{7 - An Argument for the PO Approach over Wave Function Realism based on Scientific Realism}

The above analysis also provides the PO approach with an important advantage over wave function realism. In fact, as I argue in this section, in contrast with being a realist about the PO, being a realist about the wave function may undermine the tenability of scientific realism itself in the quantum domain. Here is the reason. In the wave function ontology approach the wave function is a concrete physical field that represents matter. If we analyze this view in terms of explanationism, the wave function is a working posit of quantum theory. The problem with this is that, mathematically, the 
REVUE

DE LA SOCIÉTÉ

DE PHILOSOPHIE DES SCIENCES wave function is an object that lives in the high dimensional configuration space and as such is a very different entity from classical particles. In addition, the image of the world provided by wave function realism is very different from the image of the world given to us by classical mechanics: in the latter there are particles moving in three-dimensional space, in the former there is a matter field in a highly-dimensional space. In the classical-to-quantum transition we discover that not only we were getting the nature of objects wrong (we believed there were particles and actually there were none) but we cannot get our classical picture back by 'squinting,' like in the PO framework, since the fundamental physical space is not three-dimensional. In this way, there is no continuity of working posits between classical and quantum mechanics, and the strategy to resist to the PMI along the lines of explanationism is precluded to the proponent of wave function realism. If there is truly a quantum revolution, as wave function realism seems to maintain to a given extent (Allori 2015), and the way in which we understand the world using quantum theory is fundamentally different from the way in which we understood it in classical terms, then what is our justification to believe that the theoretical terms used in quantum mechanics are (approximately) true?

\section{ACKNOWLEDGMENTS}

I wish to thank two anonymous referees, the participants of the $6^{\mathrm{e}}$ Congrès de la Société de Philosophie des Sciences (SPS) in Lausanne, CH (June 29-July 1, 2016), the participants of the Philosophy of Science Association (PSA) meeting in Atlanta, GE (November $3-5,2016$ ), as well as the participants of the Pacific Division meeting of the American Philosophical Association (APA) in Seattle, WA (April 12-14, 2017). In particular I am grateful to Kevin Coffey for his useful comments at the Pacific APA.

\section{REFERENCES}

ALBERT, David Z. 1996. Elementary Quantum Metaphysics. In CUSHING, James, FINE, Arthur, and GOLDSTEIN, Sheldon (eds.). Bohmian Mechanics and Quantum Theory: An Appraisal. Dordrecht: Kluwer. 277-284. Link

ALBERT, David Z. 2013. Wave Function Realism. In ALBERT, David Z., and NEY, Alyssa (eds.). The Wave Function. Oxford: Oxford University Press. 52-57. Link

ALBERT, David Z. 2015. After Physics. Cambridge: Harvard University Press. Link

ALBERT, David Z., and NEY, Alyssa (eds.). 2013. The Wave Function. New York: Oxford University Press.

ALLORI, Valia, GOLDSTEIN, Sheldon, TUMULKA, Roderich, and ZANGHÍ, Nino. 2008. On the Common Structure of Bohmian Mechanics and the Ghirardi-Rimini-Weber Theory. The British Journal for the Philosophy of Science, 59, 353-389. Link

ALLORI, Valia, GOLDSTEIN, Sheldon, TUMULKA, Roderich, and ZANGHÍ, Nino. 2011. Many-Worlds and Schrödinger's First Quantum Theory. The British Journal for the Philosophy of Science, 62, 1-27. Link

ALLORI, Valia, GOLDSTEIN, Sheldon, TUMULKA, Roderich, and ZANGHÍ, Nino. 2014. Predictions and Primitive Ontology in Quantum Foundations: A Study of Examples. The British Journal for the Philosophy of Science, 65, 323-
Scientific Realism AND

Primitive Ontology

OR: The Pessimistic IN-

DUCTION AND THE NATURE OF THE WAVE FunCTION

352. Link

ALLORI, Valia. 2013. Primitive Ontology and the Structure of Fundamental Physical Theories. In: ALBERT, David Z., and NEY, Alyssa (eds.). The Wave Function. New York: Oxford University Press. 58-75. Link

ALLORI, Valia. 2015. Quantum Mechanics and Paradigm Shifts. Topoi, 32, 313-323. Link

BELL, John Stuart. 1987. Speakable and Unspeakable in Quantum Mechanics. Cambridge: Cambridge University Press. Second edition reprinted in 2004. Link

BENATTI, Fabio, GHIRARDI, GianCarlo, GRASSI, Renata. 1995. Describing the Macroscopic World: Closing the Circle within the Dynamical Reduction Program. Foundations of Physics, 25, 5-38 Link.

BHOGAL, Harjit, and PERRY, Zee. 2015. What the Humean Should Say About Entanglement. Noûs, 1, 74-94. Link

BOHM, David. 1952. A Suggested Interpretation of the Quantum Theory in Terms of 'Hidden' Variables, I and II. Physical Review, 85, 166-193. Link

CALLENDER, Craig. 2015. One World, One Beable. Synthese, 192, 3153-3177. Link

CHANG, Hasok. 2003. Preservative Realism and Its Discontents: Revisiting Caloric. Philosophy of Science, 70, 902-912. Link

DOWKER, Fay, and HERBAUTS, Isabelle. 2005. The Status of the Wave Function in Dynamical Collapse Models. Foundations of Physics Letters 18, 499-518. Link

DÜRR, Detlef, GOLDSTEIN, Sheldon, and ZANGHÍ, Nino. 1992. Quantum Equilibrium and the Origin of Absolute Uncertainty. Journal of Statistical Physics, 67, 843-907. Link DÜRR, Detlef, MÜNCH-BERNDL, Katrin, GOLDSTEIN, Sheldon, and ZANGHÍ, Nino. 1998. Hypersurface BohmDirac Models. Journal of Statistical Physics, 67, 843-907.

ELSAMAHI, Mohamed. 2005. A Critique of Localized Realism. Philosophy of Science, 72, 1350-1360. Link

ESFELD, Michael. 2017. How to Account for Quantum Non-locality: Ontic Structural Realism and the Primitive Ontology of Quantum Physics. Synthese, 194, 2329-2344. Link ESFELD, Michael, LAZAROVICI, Dustin, HUBER, Mario, and DÜRR, Detlef. 2014. The Ontology of Bohmian Mechanics. The British Journal for the Philosophy of Science, 65, 773-796. Link

ESFELD, Michael. 2004. Quantum Entanglement and a Metaphysics of Relations. Studies in History and Philosophy of Modern Physics, 35 (4), 601-617.

ESFELD, Michael. 2014. Quantum Humeanism, or: Physicalism without Properties. The Philosophical Quarterly, 64, 453-470. Link

EVERETT, Hugh. 1957. Relative State Formulation of Quantum Mechanics. Review of Modern Physics, 29, 454-462. Link

FRENCH, Stephen. 1998. On the Withering Away of Physical Objects. In CASTELLANI, Elena (ed.). Interpreting Bodies: Classical and Quantum Objects in Modern Physics. Princeton: Princeton University Press. 93-113.

GHIRARDI, Giancarlo, RIMINI, Alberto, and WEBER, Tulio. 1986. Unified Dynamics for Microscopic and Macroscopic Systems. Physical Review D, 34, 470-491. Link

GOLDSTEIN, Sheldon, and ZANGHÍ, Nino. 2013. Reality and the Role of the Wave Function in Quantum Theory. In ALBERT, David Z., and NEY, Alyssa (eds.). The Wave Func- 
REVUE

DE LA SOCIÉTÉ

DE PHILOSOPHIE

DES SCIENCES tion. New York: Oxford University Press. 96-109.

HALLIDAY, David, and RESNICK, Robert. 1962. Physics for Students of Science and Engineering, Part II. New York: John Wiley \& Sons.

KITCHER, Philip. 1995. The Advancement of Science Science without Legend, Objectivity without Illusions. Oxford University Press. Link

LADYMAN, James. 1998. What is Structural Realism?. Studies in History and Philosophy of Science, 29, 409-424. Link LADYMAN, James. 2014. Structural Realism. The Stanford Encyclopedia of Philosophy, Edward N. Zalta (ed.).

$\mathrm{URL}=\langle$ http://plato.stanford.edu/archives/spr2014/entries/structural-realism/>.

LAUDAN, Larry. 1981. A Confutation of Convergent Realism. Philosophy of Science, 48, 19-49. Link

LEWIS, Peter J. 2004. Life in Configuration Space. The British Journal for the Philosophy of Science, 55, 713-729. Link LEWIS, Peter J. 2005. Interpreting Spontaneous Collapse Theories. Studies in History and Philosophy of Modern Physics 36, 165-180. Link

LEWIS, Peter J. 2006. GRW: A Case Study in Quantum Ontology. Philosophy Compass 1: 224-244. Link

LEWIS, Peter J. 2013. Dimension and Illusion. In ALBERT, David Z., and NEY, Alyssa (eds.). The Wave Function. New York: Oxford University Press: 110-125. Link

LOEWER, Barry. 1996. Humean Supervenience. Philosophical Topics, 24(1), 101-127. Link

MAUDLIN, Tim. 2007. Completeness, Supervenience, and Ontology. Journal of Physics A, 40, 3151. Link

MAUDLIN, Tim. 2013. The Nature of the Quantum State. In ALBERT, David Z., and NEY, Alyssa (eds.). The Wave Function. New York: Oxford University Press. 126-154. Link MILLER, Elisabeth. 2014. Quantum Entanglement, Bohmian Mechanics, and Humean Supervenience. Australasian Journal of Philosophy, 92, 567-583.

MONTON, Bradley. 2002. Wave Function Ontology. Synthese, 130(2), 265-277. Link

MONTON, Bradley. 2006. Quantum Mechanics and 3-N Dimensional Space. Philosophy of Science, 73 (5), 778-789.

HISTORIQUE

Article initialement soumis le 31 octobre 2016.

Version révisée soumise le 13 septembre 2017.

Article accepté le 15 septembre 2017.

SITE WEB DE LA REVUE

sites.uclouvain.be/latosensu/index.php/latosensu/index

ISSN 2295-8029

DOI HTTP://DX.DOI.ORG/10.20416/LSRSPS.V5I1.10

\section{SCIENTIFIC REALISM AND \\ Primitive Ontology \\ Or: The Pessimistic Induc- TION AND THE NATURE OF THE WAVE FunCTION}

Link

NEY, Alyssa. 2012. The Status of Our Ordinary Three-dimensions in a Quantum Universe. Noûs, 46, 525-560. Link

NEY, Alyssa. 2013. Ontological Reduction and the Wave Function Ontology. In ALBERT, David Z., and NEY, Alyssa (eds.). The Wave Function. New York: Oxford University Press, 168-183. Link

NEY, Alyssa. 2015. Fundamental Physical Ontologies and the Constraint of Empirical Coherence. Synthese, 192(10), 3105-3124. Link

NEY, Alyssa. 2017. Finding the World in the Wave Function: Some Strategies for Solving the Macro-object Problem. Synthese, 1-23. Link

NEY, Alyssa. Forthcoming. Separability, Locality, and Higher Dimensions in Quantum Mechanics. In DASGUPTA, Shamik, and WESLAKE, Brad, (eds.). Current Controversies in Philosophy of Science. London: Routledge.

NORSEN, Travis. 2010. The Theory of (Exclusively) Local Beables. Foundations of Physics 40, 1858-1884. Link

NORTH, Jill. 2013. The Structure of the Quantum World. In ALBERT, David Z., and NEY, Alyssa (eds.). The Wave Function. New York: Oxford University Press, 184-202. Link

PSILLOS, Stathis. 1994. A Philosophical Study of the Transition from the Caloric Theory of Heat to Thermodynamics: Resisting the Pessimistic Meta-Induction. Studies in History and Philosophy of Science, 25, 159-90. Link

PSILLOS, Stathis. 1999. Scientific Realism: How Science Tracks Truth. London: Routledge. Link

STANDFORD, Kyle. 2003a. Pyrrhic Victories for Scientific Realism. Journal of Philosophy, 100, 553-572.

STANDFORD, Kyle. 2003b. No Refuge for Realism: Selective Confirmation and the History of Science. Philosophy of Science, 70, 913-925. Link

TUMULKA, Roderich. 2006. A Relativistic Version of the Ghirardi-Rimini-Weber Model. Journal of Statistical Physics, 125, 821-840. Link

WORRALL, John. 1989. Structural Realism: The Best of Both Worlds? Dialectica, 43, 99-124. Link

\section{CONTACT ET COORDONNÉES}

Valia Allori

Department of Philosophy, Northern Illinois University Zulauf Hall 915,

Dekalb IL 60115, USA

vallori@niu.edu 